Article

\title{
Electrical Detection of Degradation in Specimens of HVDC Cable Insulation ${ }^{+}$
}

\author{
Douglas Jutsell Nilsson *, Stanislaw M. Gubanski * (i) and Yuriy V. Serdyuk *(1) \\ Department of Electrical Engineering, Chalmers University of Technology, 41296 Gothenburg, Sweden \\ * Correspondence: jutsell@chalmers.se (D.J.N.); stanislaw.gubanski@chalmers.se (S.M.G.); \\ yuriy.serdyuk@chalmers.se (Y.V.S.) \\ + This paper is an extended version of a conference paper, Proceedings of the Nordic Insulation Symposium \\ NORD-IS19, Tampere, Finland, 12 June 2019.
}

Received: 1 July 2020; Accepted: 27 July 2020; Published: 1 August 2020

\begin{abstract}
One of the challenges in laboratory investigation of degradation and ageing of HVDC cable insulation is related to securing, or in other words, imitating the real service environment of the material specimens. So far, the published data refer to experiments conducted in thermo-oxidative conditions, which is not the case during normal cable operation. In service, the cable insulation is protected by a metallic barrier that blocks the transfer of any substances in and out of the construction. By-products from the cross-linking reactions cannot diffuse out and any foreign substances are blocked from entering the insulation. Thus, in order to generate results that are valid, these conditions must be replicated in laboratory experiments. This contribution presents a methodology elaborated for performing ageing experiments in a hermetically sealed environment. Degradation of the material is evaluated through changes in the electrical tree inception voltage and test object capacitance over time. Securing the environmental isolation is accomplished with an isolation system consisting of a glass enclosure with attached metallic electrodes. Indium is used to create a glass-to-metal seal between the glass and the electrodes. The electrode geometry is of needle-plane type and the needle injection process is semi-automated to ensure specimen repeatability.
\end{abstract}

Keywords: electric tree; HVDC-cable insulation ageing; XLPE ageing; electric tree capacitance

\section{Introduction}

Understanding the effects on HVDC cable insulation caused by long-term exposure to in-service stresses is key to evaluating current and emerging materials. Experiments which yield such results can be carried out in a number of different ways, for example, by conducting full-scale tests using actual or model cables, by using cables that have already been in-service or by carrying out laboratory experiments on miniature specimens with artificial defects. Full scale experiments, and to a certain extent, model cables, are costly endeavors. Miniature specimens, of a size such that they fit in the palm of a hand, taken from an already produced cable can offer significant advantages. The voltage required for experimentation on this scale is typically in the lower range of tens of kilovolts, as opposed to the hundreds of kilovolts required for the full-scale tests. For this reason, the equipment and space requirements are manageable and miniature specimens with artificial defects present a tempting economical alternative. Furthermore, a specimen from an already produced cable ensures that there are no differences between the examined material and the insulation in-service. The downside arises; however, when trying to relate the stress level introduced by the defect to that of the stresses found in an actual cable.

There are two weakly connected issues that complicates the effort of reproducing ageing of HVDC cable insulation. They are linked to the properties of extruded cross-linked polyethylene, which is 
currently the prevailing material used for these applications [1]. The first important matter is the possible injection and transport of charge carriers, which influences the electric field distribution. The second relates to the metallic sheath that surrounds a typical cable in-service. The cross-linking process leaves residual by-products in the insulation which has a large effect on the electrical properties [2]. Thus, the electric conductivity and space charge formation are strongly dependent on their concentration levels [3]. Moreover, electrical tree inception and subsequent breakdown are also dependent on the concentrations of residuals when the excitation voltage is sinusoidal and of low frequency. At impulse voltages, the effect of by-product is less significant [4].

Since the insulation in an actual cable is surrounded by a metallic sheath, the by-products are naturally contained within the material. The metallic sheath also serves to block any foreign matter from entering the insulation. A substantial amount of research exists showing the debilitating results obtained for the material immersed in atmospheric air where oxygen is available causing material degradation that intensifies with increasing temperature [5-8]. Even low levels of oxygen have been shown to have a negative effect on the additives that are responsible for tree suppression in the insulation. Instead of reacting with free radicals in the high-field region, the additive reacts with oxygen which leads to rapid chain scission and electric tree growth [9]. In a cable with a metallic sheath, these conditions are simply not present, instead the oxygen content is found to decrease with time due to the presence of anti-oxidants [6].

Additional effects introduced in traditional measuring methodologies for studying insulation ageing are related to presence of moisture and elevated temperatures. It has been shown that high moisture content leads to an increase in the dissipation factor, a decrease of the volume resistivity and a loss of the dielectric strength [10]. Regarding temperature, in electrical treeing experiments where a static sinusoidal voltage is used, the inception time has been shown to decrease significantly when the temperature is raised from 25 to $90^{\circ} \mathrm{C}$. This effect becomes weaker with higher excitation voltages [4]. In experiments conducted with artificial defects in the range of 5 to $20 \mu \mathrm{m}$ and ramped voltages, the inception voltage for electrical trees was decreased by $15-30 \%$ when the temperature was raised from 20 to $90{ }^{\circ} \mathrm{C}$. For the same temperature range but under impulse voltage excitation, the inception voltage was lowered by $66 \%$ [11].

The analysis above indicates that ageing of cable insulation is affected by many factors both external and internal. However, the former can be considered as stresses acting on the insulation whereas the latter reflect conditions inside the cable. Therefore, for ageing experiments to be valid, the actual environmental conditions of the insulation must be replicated. This contribution presents a methodology elaborated for performing ageing experiments in a hermetically sealed environment based on measurements of capacitances of the material samples during development of electrical trees.

\section{Material Samples Preparation and Measuring Methods}

\subsection{Material Samples}

Material specimens have been taken from an actual HVDC cable that was stored with the ends sealed by metallic foil. The cable originates from a bipolar link operated at $\pm 300 \mathrm{kV}$. Specimens were taken radially with a final height of $17 \mathrm{~mm}$ and a base of $10 \times 10 \mathrm{~mm}$ (about $1 \mathrm{~mm}$ of the inner semiconductor was present). To protect the material from environmental factors, the specimen was encapsulated as shown in Figure 1. As seen, the cuboid specimen is located in between two brass electrodes, inside a glass tube with a square cross-section. The transparent blueish part in Figure $1 \mathrm{~b}$ indicates the polymer and the black part represents the inner semiconductor layer. An artificial defect in the form of a needle in contact with one of the electrodes was used to create a small volume of high electric field strength around its tip. The distance between the semiconductor and the tip was $4 \mathrm{~mm}$ and the tip diameter was $5 \pm 0.5 \mu \mathrm{m}$. The main property of the specimen capsule is its ability to hinder interaction between the specimen and the outside world while still maintaining electrical contact with the semiconductor and the needle electrode. This is accomplished by using an arrangement of 
glass and brass. The use of glass is motivated by the fact that it is one of the few materials that is transparent, has excellent dielectric properties and that any permeation through it should be negligible for this application.

A glass-to-metal joint is utilised to seal the capsule hermetically. The seal itself is a multi-step process involving indium, a chemical element that wets and bonds against glass and that has a low melting temperature. The element is commonly used when there is a need to join non-metallic materials to other non-metallic materials or metals.

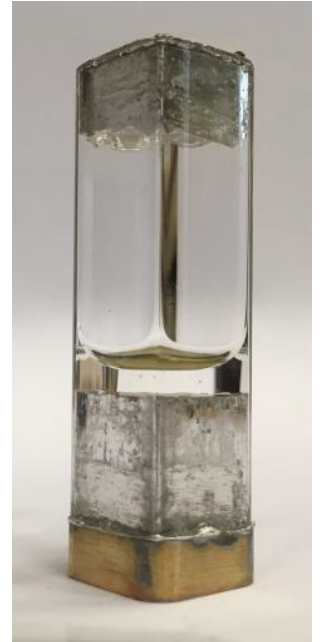

(a)

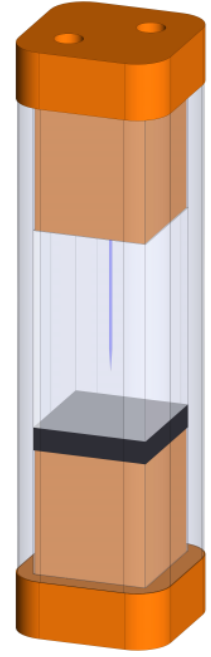

(b)

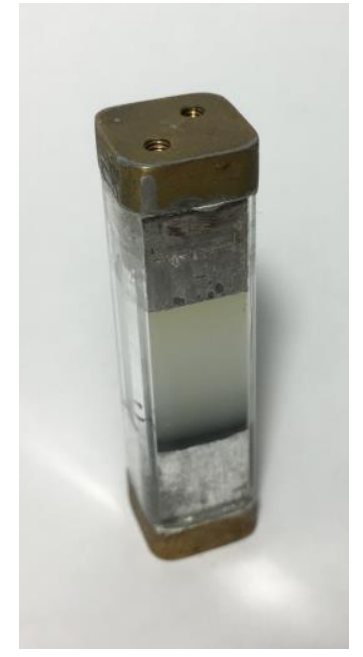

(c)

Figure 1. (a) The specimen capsule during assembly; (b) the geometry of the specimen capsule; and (c) the finished specimen capsule.

\subsection{Needle Injection and Polymer Processing}

The needle injection process has substantial implications on the repeatability of the experiments. The way it is carried out influences the final geometry of the specimen, the amount of cross-linking by-products in the specimen, and the polymeric composition of the final specimen. The injection process involves three steps:

- Heating the specimen to a point where the polymer is completely amorphous.

- Inserting the needle while the temperature is kept stable.

- Cooling the specimen back down to room temperature with the needle residing in it.

The implications for repeatability, and a part of the difficulty in this task, comes from the fact that the polymer expands when it is heated and contracts when cooled. Furthermore, the final volume of the polymer is determined by the rate of cooling. The longer time it is given to cool, the more time the specimen has to form crystalline regions. These have a higher density than the amorphous ones and thus, occupies less volume. This implies that in order to ensure that different specimens have the same degree of crystallinity, the temperature curve that the specimens are exposed to during injection must be almost identical, with an emphasis on the rate of cooling. The rate of loss of the cross-linking by-products from the specimen naturally also increases with temperature, implying a need for a closed environment when the specimen is heated. Further, thermo-oxidation will occur at amorphous temperatures where the antioxidant additives will be consumed, should they be depleted, chain scission due to oxidation will start to occur [5-8].

A design that accommodates for the above realities is shown in Figure 2a. In Figure $2 b$ the actual device is seen. The specimen is completely surrounded by metal. The piece that is located on top of the specimen is similar to the electrode used in the capsule but made from two separate pieces in this case. Each one has a groove engraved that is just slightly larger than the needle. The two 
parts are held together with screws. The grooves line up and allows the needle passage through the part. Mechanically, it is held down by four screws, keeping it in place as the specimen tries to expand. The angled orange part in the figure is a thin sheet of copper that is used to try and reduce the leakage from the injection cell. It is meant to deform and seal when pressure is applied. Heating elements are screwed to the sides of the specimen to facilitate heating and the temperature is regulated by a PI-controller fed with a predetermined temperature cycle. A chuck capable of centring the needle is mounted on a linear rail and controlled by a computer, allowing precise control over the needle injection.

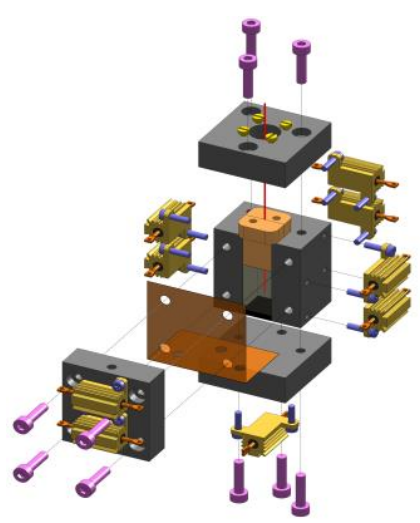

(a)

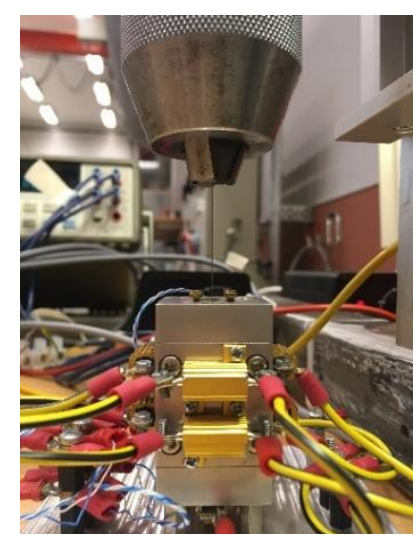

(b)

Figure 2. (a) Geometry of the injection cell; (b) the finished setup.

The temperature cycle that was applied during the injection process is shown in Figure 3. The set point arcs for the heating and the beginning of the cooling sections are parametrised circles in order to avoid exposing the PI-regulator to step changes in the set point. The first mark in the figure indicates where the needle starts to be lowered, the second when it starts to penetrate the polymer, and the third when it has reached its final depth.

Before the actual injection process is started, the needle is referenced to the top of the polymer and then raised above the top piece of the injection cell. This is done to avoid any potential interaction between the tip of the needle and any eventual polymer that enters the injection channel during the heating up of the cell.

The final geometry of the specimen is dependent on the injection procedure. When cutting the specimens from a cross-section of the cable, it is not practical to try to ensure a perfect cuboid, especially since the shape of the specimen inevitably is going to be altered to some degree by going through the injection process later. Using this injection cell with the temperature curve as seen in Figure 3 produces specimens which shape is that of the inner dimensions of the injection cell down to the surface texture of the machined metal parts. A minute loss of density has thus occurred, but the compromise is well worth it since it enables the production of almost geometrically identical specimens. 


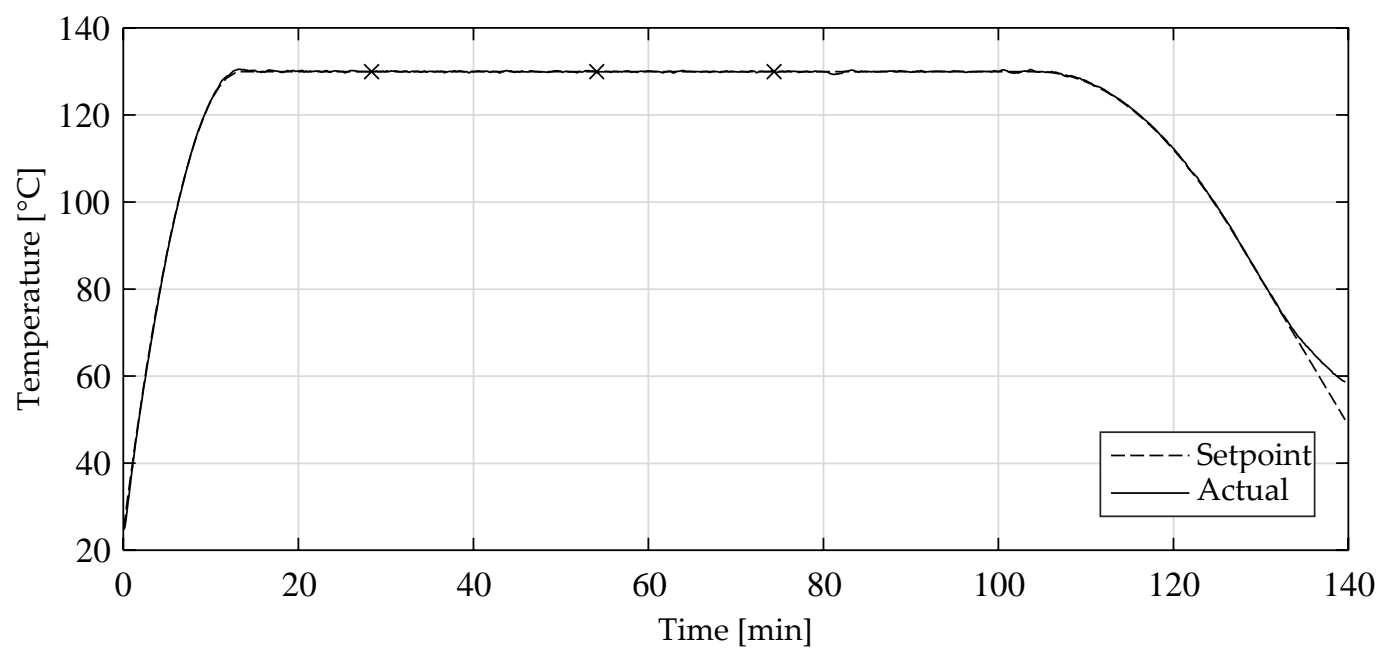

Figure 3. Temperature during the needle injection process.

\subsection{The Test Cell}

In the experiments, the specimen capsule was placed in a test cell as shown in Figure 4. The cell provided electric field control in order to reduce the otherwise high electric fields that would develop around the sharp edges of the electrodes. Further, it served to constrain the capacitance of the specimen. An important requirement of the electrode system was that its measured capacitance should be as small as possible to better resolve small capacitive changes in the volume in close vicinity of the tip of the needle. Additionally, the specimen capsule should be fixed in place in order to keep it from experiencing any change in its capacitive environment. The cell supports the specimen capsule axially as there is no need to stress the glass-to-metal seal unnecessarily and finally, the needle tip is optically observable.

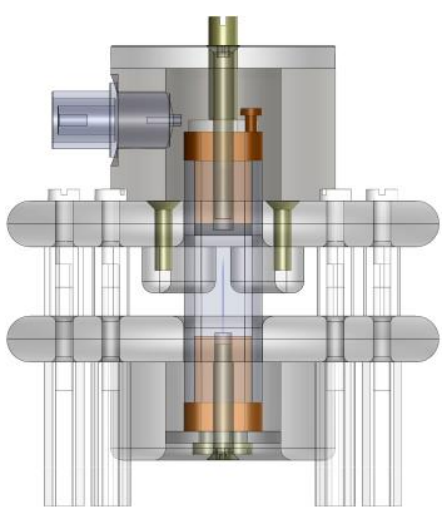

(a)

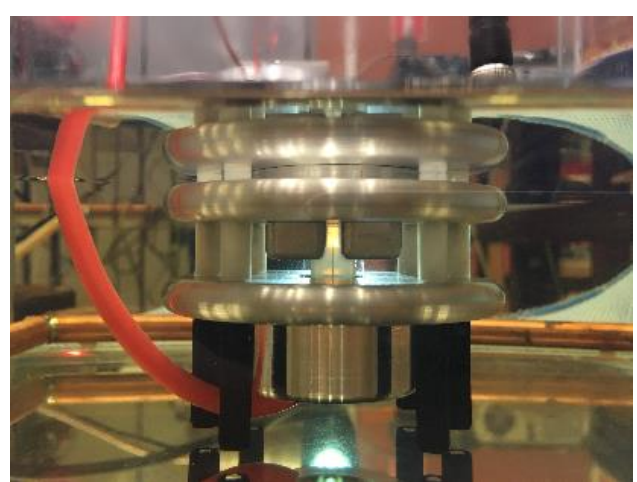

(b)

Figure 4. (a) A cross section of the cell geometry; (b) the finalized cell, submerged in oil.

Ultimately, the cell was submerged in mineral oil. The oil was used both as a dielectric and as a heat transfer medium. It was necessary since the distance at the narrowest part of the cell between the two polarities was $4 \mathrm{~mm}$ which ruled out keeping the cell in air during electrical tree inception experiments. The temperature in the bath was regulated using heating elements controlled by PI-controller.

\subsection{The Reference Impedance}

A known impedance connected in series with the test object allows changes in the specimen impedance to be quantified accurately. The impedance consists of a parallel resistive-capacitive 
arrangement of $1 \mathrm{M} \Omega \| 112 \mathrm{pF}$, Figure 5a. The output voltage, i.e., the voltage developed over the impedance, is buffered by an operational amplifier. Present is also an amplifier that actively drives a guard. The choice of a resistive-capacitive impedance was motivated by two factors:

- The resistive part facilitates usage with both $\mathrm{AC}$ and $\mathrm{DC}$ voltages.

- If there is PD-activity in the specimen, the capacitor integrates the PD-signal, making the otherwise transient PD-events visible when measuring at relatively low sampling frequencies.

The component values were chosen based on approximations of the specimen impedance. Given that the specimen and the reference impedance form a voltage divider, the values of the components must be such that the voltage developed over the reference impedance does not exceed the supply voltage of the buffering amplifier. Furthermore, the RC-time constant should be appropriate for the sampling frequency.

A femtoampere level operational amplifier operating in unity gain mode was used to buffer the signal. The low bias current enabled measurements of high impedance specimens without skewing the results. The amplifier also isolated the impedance from any influence of connected data acquisition systems. The output of the amplifier acted as the input for the guard driver, which fed the guard traces on the printed circuit board, Figure $5 \mathrm{~b}$. The guard continues in the shield of the cable connecting the reference impedance to the cell, either via the outer shield of a coaxial cable or the inner shield of a triaxial cable.

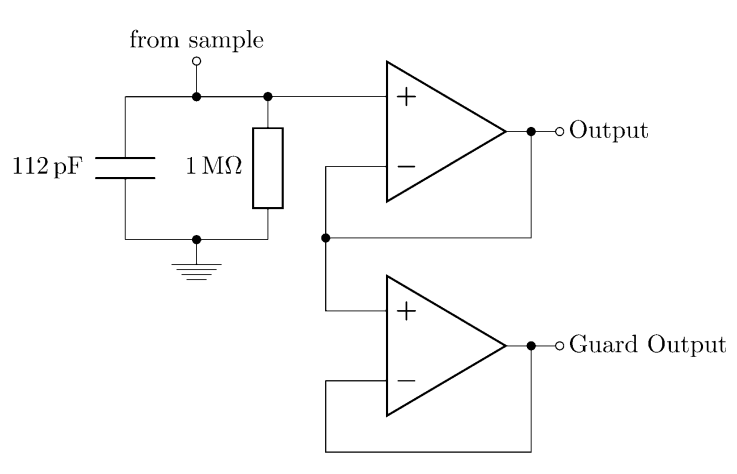

(a)

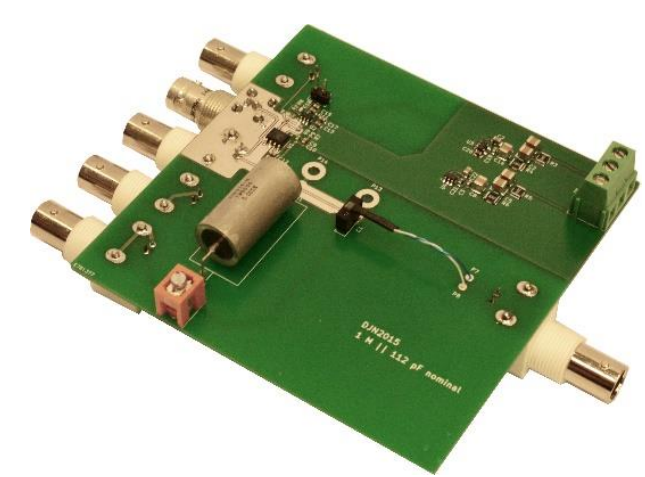

(b)

Figure 5. (a) A simplified schematic of the reference impedance; (b) the actual device.

The buffer amplifier was a LMP7721 while the guard was driven by a LMP7715. The passive components were selected mainly based on their long-term stability. The resistive part was provided by a Vishay VHA516-6 resistor, an oil filled, hermetically sealed secondary standard resistor, whose bulk metal foil construction gives it low values of parasitic inductance and capacitance. The resistor exhibits a TCR of $\pm 2 \mathrm{ppm} /{ }^{\circ} \mathrm{C}$ and a load life stability of $\pm 0.005 \%$. The tolerance of the nominal value is $\pm 0.001 \%$.

A capacitor of type AVXM23269J/10 3094, constructed of a metallic structure embedded in glass and sealed hermetically, provided $100 \mathrm{pF}$ of the capacitive part of the impedance (the remaining $12 \mathrm{pF}$ was contributed by the input capacitance of the LMP7721-amplifier). According to the manufacturer, the capacitor exhibits "zero ageing" and a TCC of $100 \mathrm{ppm} /{ }^{\circ} \mathrm{C}$. Due to this, the reference impedance circuit board was installed in a temperature regulated enclosure. The temperature of the housed reference impedance was recorded for two weeks and the results are shown in Figure 6. As seen, the inner temperature was held within $30.3^{\circ} \mathrm{C}$ and $29.8^{\circ} \mathrm{C}$. 


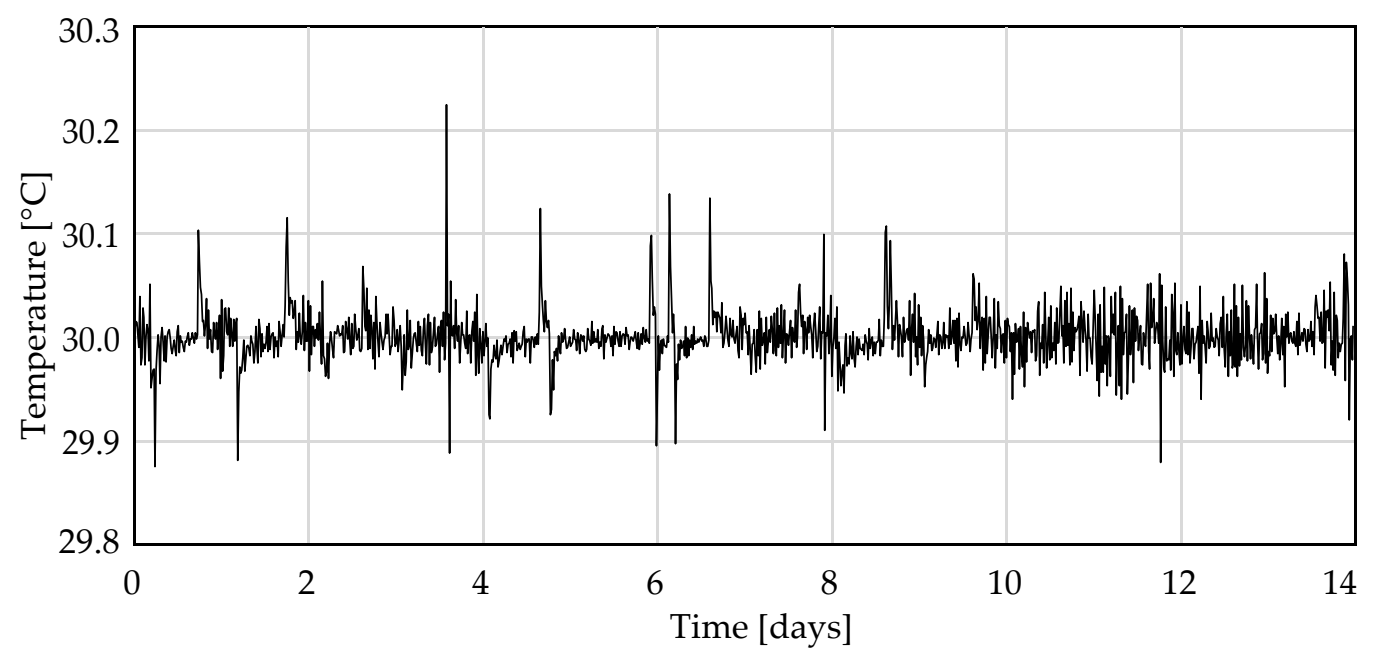

Figure 6. Temperature of the reference impedance for two weeks.

\subsection{Setup for Electric Tree Inception Voltage Measurements}

Measurements of the inception voltages of electrical trees were carried out using the system shown in Figure 7. It consisted of the reference impedance connected in series with the specimen, a voltage divider, the submerged test cell as well a conventional PD-measuring circuit: a coupling capacitor and a coupling quadripole. It was controlled by computers that were responsible for supervising two temperature control loops (the reference impedance and the oil bath), the recording of data from digital acquisition devices, the generation of signals using digital-to-analogue converters, and exercising control of the setup in conjunction with various instruments and devices. A summary of the instrumentation list for the setup is found in Table 1.

As the setup is meant to be used to terminate the ageing process of a specimen by measuring the inception voltage of an electrical tree, an inherently destructive event for the specimen, the generated data point of the inception voltage is quite precious. Its value originates from the time involved ageing, the effort spent preparing the specimen, injecting the needle, and sealing the capsule etc. Because of this, the data acquisition system was configured to record a continuous stream of data from each source and save them for later analysis.

Table 1. A summary of the devices in use.

\begin{tabular}{ccc}
\hline Device Type & Name & Configuration \\
\hline DAQ & NI-4431 & 24 bits, $\pm 10 \mathrm{~V}, \mathrm{f}=102.4 \mathrm{kHz}$ \\
DAQ & NI-5133 & 8 bits, $\pm 4 \mathrm{~V}, \mathrm{f}=10 \mathrm{MHz}$ \\
DAC & NI-6153 & 16 bits \\
PD-calibrator & Haefly type 451 & \\
PD-coupling & Haefly akv type 568 & $20 \mathrm{~mA}, 1 \mathrm{nF}$, amplifier: on \\
quadripole & Haefly & $1000 \mathrm{pF}, 75 \mathrm{kV}$ \\
PD-coupling & Olympus SZ61 & Camera: Olympus SC30 \\
capacitor & &
\end{tabular}

The system makes four cardinal sources of data available for analysis:

- the voltage applied to the test object, measured by a NI-4431;

- $\quad$ the voltage developed over the reference impedance, measured by the NI-4431;

- the voltage output from the quadripole, measured by a NI-5133; and

- the video stream capturing the tip of the needle, recorded by a camera through a microscope. 
Having access to the entire waveform produced from all data acquisition sources also enables precise analysis of the behaviour of the specimen up to and after the inception of an electrical tree, further elucidating the behaviour of the specimen examined.

The excitation waveform was a ramped sinusoidal signal. The waveform was generated by a digital-to-analogue converter, amplified by a class $\mathrm{AB}$-amplifier, and fed to a high voltage transformer.

The frequency of the applied waveform was $55.71 \mathrm{~Hz}$ and the ramp rate was $12 \mathrm{~V}_{\mathrm{rms}} / \mathrm{s}$. At the start of the measurement, the various data acquisition systems were synchronised time-wise.

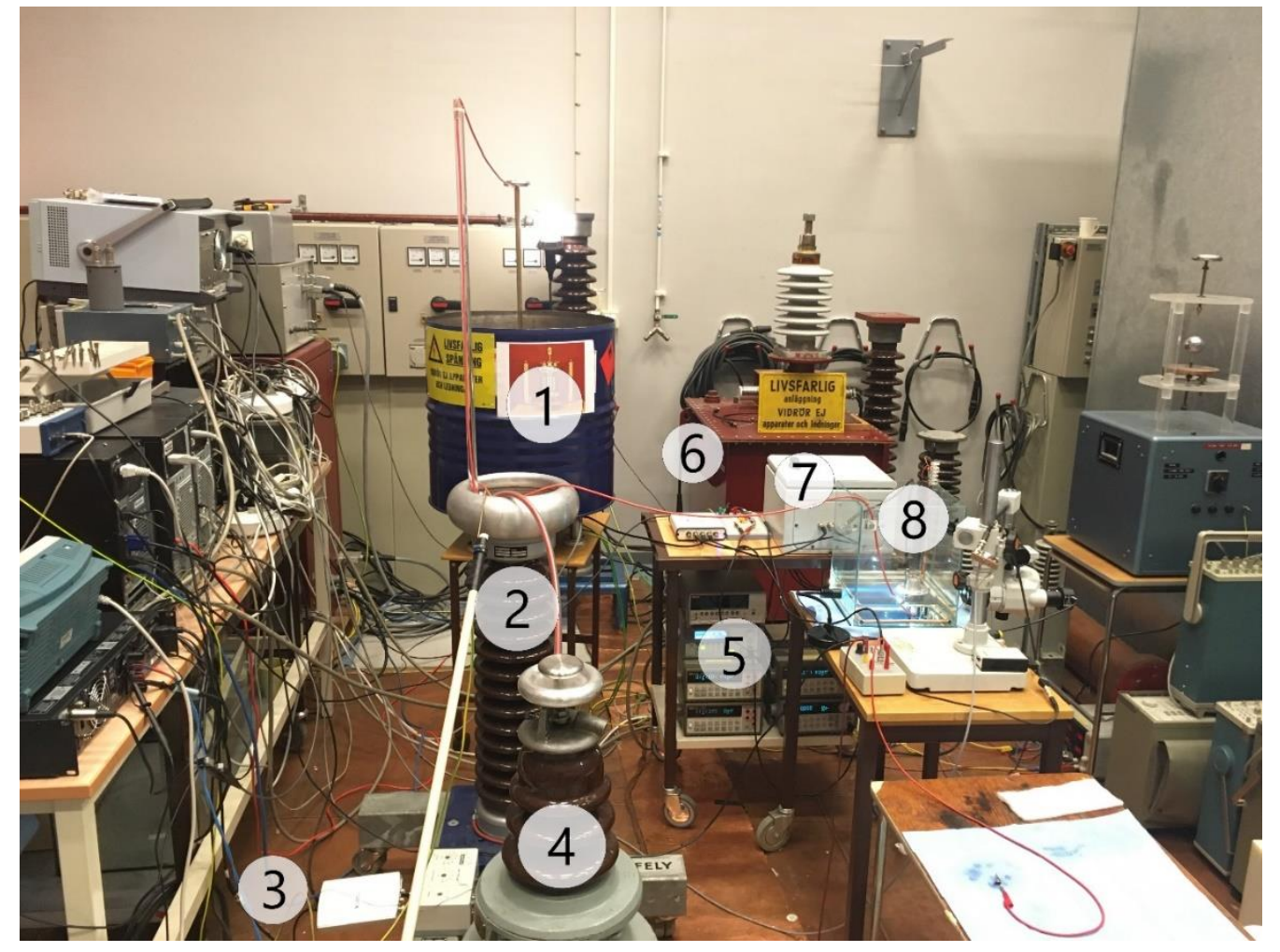

Figure 7. The setup which facilitated the electrical tree inception experiments. (1) Voltage divider, (2) coupling capacitor, (3) NI-5133, (4) transformer, (5) signal generation and RTD-measurement, (6) NI-4431, (7) reference impedance, and (8) test cell submerged in oil.

\section{Results}

A comparison between the detected tree inception voltage level across the available data sources of an experiment is shown in Table 2.

Table 2. Time and voltage of detected inception from the different data sources.

\begin{tabular}{ccc}
\hline Source & Time of Detection & RMS Voltage $[\mathbf{k V}]$ \\
\hline Uref-imp & $17 \mathrm{~m} \mathrm{37.37}$ & \\
PD & $17 \mathrm{~m} \mathrm{37.42} \mathrm{s}$ & 12.62 \\
Optical & $12 \mathrm{~m} \mathrm{37.28 \textrm {s }}$ & \\
\hline
\end{tabular}

Although the inception voltage can be accurately detected by any of the sources, as is evident by Table 2, further understanding of what transpires in a specimen during an electrical treeing can be gained from calculating the impedance of the specimen during the experiment.

A schematic of the specimen, (modelled as an RC-parallel circuit), connected to the reference impedance is shown in Figure 8. 


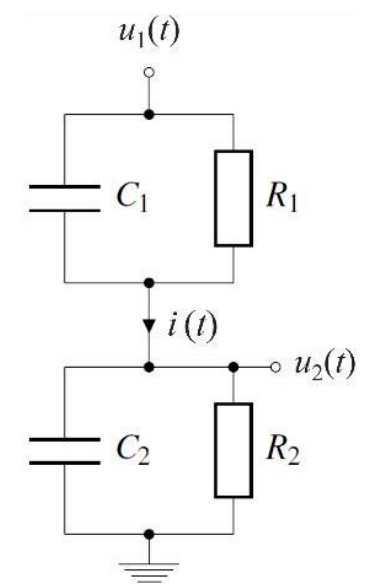

Figure 8. Schematic representation of the device under test connected to the reference impedance.

The current in the circuit can be expressed as

$$
i(t)=\frac{u_{1}(t)-u_{2}(t)}{R_{1}}+C_{1} \frac{\mathrm{d}\left(u_{1}(t)-u_{2}(t)\right)}{\mathrm{d} t}=\frac{u_{2}(t)}{R_{2}}+C_{2} \frac{\mathrm{d} u_{2}(t)}{\mathrm{d} t}
$$

where $u_{1}$ is the applied voltage to the specimen and $u_{2}$ is the voltage over the reference impedance, both of which are measured quantities. $R_{1}$ and $C_{1}$ are the values of the model components of the specimen and $R_{2}$ and $C_{2}$ the values of the reference impedance.

Equation (1) was solved numerically. In order to find $R_{1}$ and $C_{1}$, multiple regression method was used and the fit was dependent on more than one predictor variable. Specifically, the circuit was modelled by

$$
A_{1} x_{1}+A_{2} x_{2}=B
$$

where $A_{1}=u_{1}(t)-u_{2}(t), x_{1}=1 / R_{1}, A_{2}=\mathrm{d}\left(u_{1}(t)-u_{2}(t)\right) / \mathrm{d} t, x_{2}=C_{2}$ and $B=i(t)$. Solving $\mathbf{A} \mathbf{x}=\mathbf{B}$ for $\mathbf{x}$ was carried out by the Matlab command "mldivide", which uses QR decomposition to find a least-squares solution of the equation system thus yielding $R_{1}$ and $C_{1}$. By calculating the impedance in this way, the influence of PD-activity on the results was lessened.

With all circuit parameters known, the discharge free voltage $u_{2}$ based on the excitation voltage $u_{1}$ was calculated using Heun's method, which (with the same electrical notation as before) resulted in the expression

$$
u_{2}(t+h)=u_{2} \frac{1}{2}\left(k_{1}+k_{2}\right),
$$

where $k_{1}=h \cdot \mathrm{d} u_{2}(t) / \mathrm{d} t$ and $k_{2}=h \cdot \mathrm{d} u_{2}(t+h) / \mathrm{d} t$. Note that $k_{1}$ represents the derivative multiplied by the step length $h$ at the time $t$ and $k_{2}$ is the derivative of $u_{2}$ at $t+h$ multiplied by $h$. The former is readily calculated by rearranging Equation (1), as shown in Equation (4). The latter expression is seen in (5) and was obtained based on the Euler method for an approximation of $u_{2}(t+h)=u_{2}(t)+k_{1}=$ $u_{2}(t)+h \cdot \mathrm{d} u_{2}(t) / \mathrm{d} t$.

$$
\begin{gathered}
\frac{\mathrm{d} u_{2}(t)}{\mathrm{d} t}=\frac{\frac{u_{1}(t)}{R_{1}}+C_{1} \frac{u_{1}(t+h)-u_{1}(t-h)}{2 h}-u_{2}(t)\left(\frac{1}{R_{1}}+\frac{1}{R_{2}}\right)}{\left(C_{1}+C_{2}\right)}, \\
\frac{\mathrm{d} u_{2}(t+h)}{\mathrm{d} t}=\frac{\frac{u_{1}(t+h)}{R_{1}}+C_{1} \frac{u_{1}(t+2 h)-u_{1}(t)}{2 h}-\left(u_{2}(t)+k_{1}\right)\left(\frac{1}{R_{1}}+\frac{1}{R_{2}}\right)}{\left(C_{1}+C_{2}\right)} .
\end{gathered}
$$

The differentiation of $u_{1}$ was kept as a finite difference in order to avoid having a 0.5 sample offset and the step length $h$ was equal to the time between samples. 
For the initial value problem (4) and (5), a starting value for $u_{2}$ must be chosen. One way is to set it to zero and allow for the approximation to settle, but the length of time before that occurs depends on the waveform window. The worst-case scenario, when the window begins with one of the maximum or minimum peaks, requires $\sim 60$ samples to be processed before the approximation settles. An improvement can be made by using the actual sample of $u_{2}$ at the beginning of the window, but this can be catastrophic if a PD occurs at that sample in time. A practical way of finding a suitable initial value is to use a fitted curve resulting from a trigonometric Fourier series fit in the form of

$$
y=a_{0}+a_{1} \cos (\omega x)+b_{1} \sin (\omega x)
$$

With the help of Matlab's curve fitting toolbox, the curve was fitted in a non-linear least square sense using a trust-region algorithm, yielding $\omega, a_{0}, a_{1}$, and $b_{1}$. This method allows for $u_{2}$ to be calculated for any given time window of the waveform, which in turn enables the discharge free current to be calculated using the same expression as before (the right side of (1)), but with the approximated voltage $u_{2}$ instead of the sampled $u_{2}$.

Figures 9-11 show an example of the results of the calculations from an electrical treeing experiment. Here, the computation was conducted on a one-period basis. Figures 9 and 10 show the same timeframe of 1.5 periods from an experiment just after that of the inception of an electrical tree. The measured current, the waveform that the calculated impedance of the specimen would have produced given the measured excitation voltage as well as the difference between the two are presented in Figure 9. The voltage measured over the reference impedance as well as the numerical approximation of the expected voltage (developed given the calculated impedance of the specimen) are provided in Figure 10. Finally, Figure 11 shows the calculated capacitance of a specimen over a timeframe that begins just before the inception of an electrical tree and during one minute of growth.

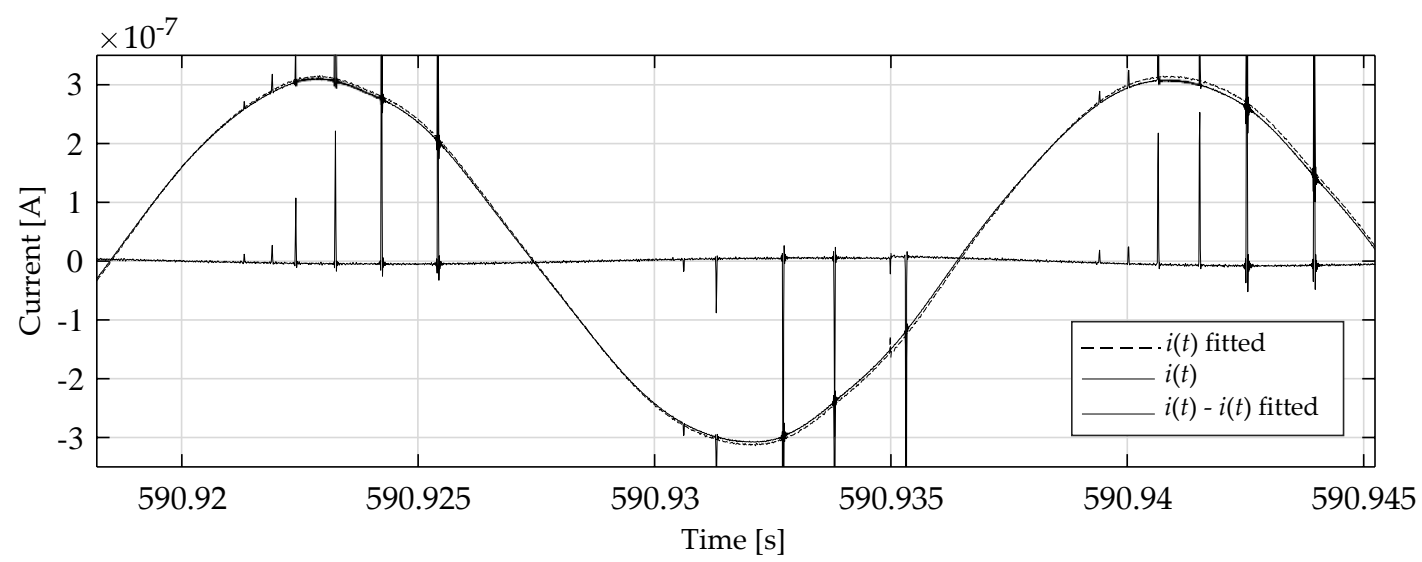

Figure 9. The actual current as well as the discharge free current from the regression calculation current during 1.5 periods of an experiment after the inception of an electrical tree. 


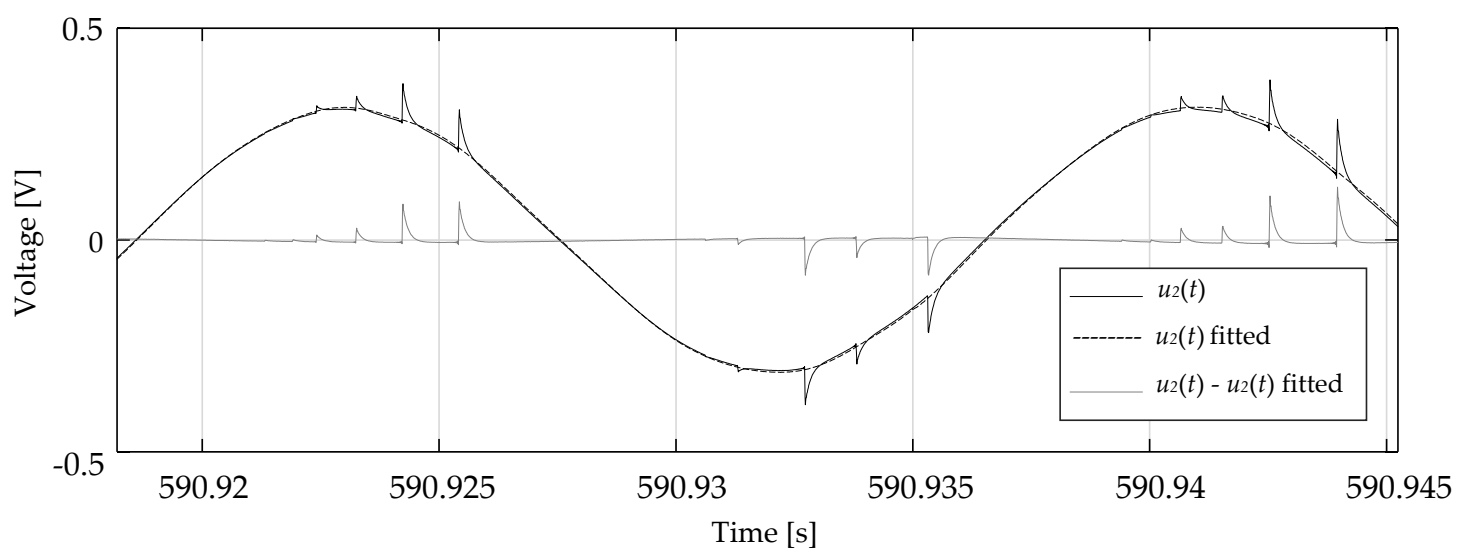

Figure 10. The actual voltage $u_{2}$ as well the fit resulting from the regression calculation and Heun's method during 1.5 periods of an experiment after the inception of an electrical tree.

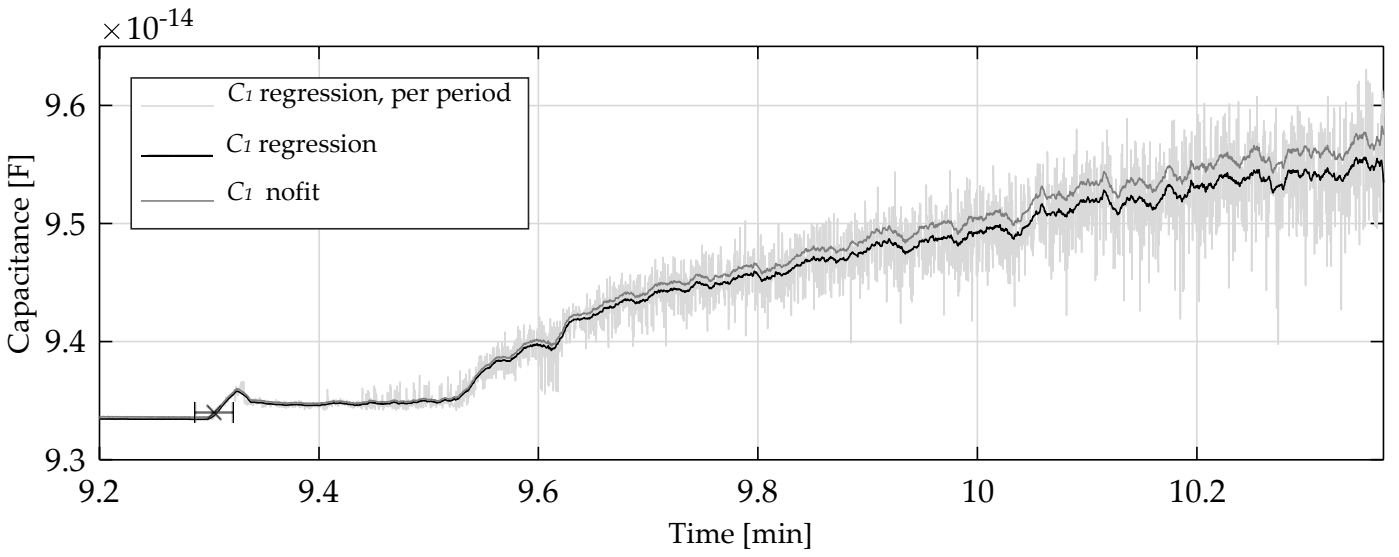

Figure 11. A window of the capacitance during electrical tree growth. The mark indicates where the electrical tree was detected optically. " $C_{1}$ regression" and " $C_{1}$ nofit" are results of rolling average of 43 periods. Nofit refers to a simple jw-calculation where no effort has been made to lessen the influence of the ongoing PD-activity on the capacitance.

\section{Conclusions}

The overall goal of the presented work was to develop measuring methodology which would allow (i) to locate material samples in an environment that resembles an actual HVDC-cable and (ii) to detect the tree inception voltage during an experiment at increasing applied sinusoidal voltage. The first goal can be considered to have been met. The current method of determining the success of a finalised specimen capsule is to submerge it in liquid and conducting a bubble test, visually checking for leakage to test the hermeticity of the seal. While this does not quantify any leakage in numbers, it is a substantial step in the right direction. Given the results presented, the second goal has been met successfully as every source of detection yielded an accurate value of the electrical tree inception voltage.

Out of the available detection sources, the signal from the reference impedance is arguably both the most practical and informative measure. It can be used to calculate the impedance of the specimen in which the electrical tree inception voltage is easily detected as it manifests as a distinct rise in capacitance. It also contains the base capacitance of the specimen as well as the capacitance change per time unit during tree growth. Both latter quantities are useful for electrical treeing experiments since they can be used as an indicator of the repeatability of the specimens. Finally, it provides access to the current in the circuit, which is by far the best quantity to look at to get a quick overview of the outcome of an experiment. 
Thus, the methodology described in this work, with an emphasis on the needle injection procedure and the specimen capsule, is ready for an evaluation on a larger scale. A large series, in the range of 40 specimens, is currently being prepared.

Author Contributions: Investigation, D.J.N.; writing—original draft, D.J.N.; writing—review and editing, S.M.G. and Y.V.S.; supervision, S.M.G.; and funding acquisition, S.M.G. All authors have read and agreed to the published version of the manuscript.

Funding: This project was funded by Svenska Kraftnät. The project also acknowledges a grant from Fond till minne av Erik Feuk, (SC 2015-0036).

Conflicts of Interest: The authors declare no conflict of interest.

\section{References}

1. Reed, C.W. An assessment of material selection for high voltage DC extruded polymer cables. IEEE Electr. Insul. Mag. 2017, 33, 22-26. [CrossRef]

2. Andrews, T.; Hampton, R.N.; Smedberg, A.; Wald, D.; Waschk, V.; Weissenberg, W. The role of degassing in XLPE power cable manufacture. IEEE Electr. Insul. Mag. 2006, 22, 5-16. [CrossRef]

3. Shinoda, C.; Hashizume, T.; Tani, T.; Tanaka, Y.; Takada, T. A consideration of mechanism of DC leakage current peak in XLPE cables. In Proceedings of the 5th International Conference on Properties and Applications of Dielectric Materials, Seoul, Korea, 25-30 May 1997; Volume 1, pp. 402-405. [CrossRef]

4. Densley, R.J. An Investigation into the Growth of Electrical Trees in XLPE Cable Insulation. IEEE Trans. Electr. Insul. 1979, EI-14, 148-158. [CrossRef]

5. Gubanski, S.M.; Karlsson, K.; Gedde, U. Study of thermal oxidation in medium density polyethylene. In Proceedings of the IEEE International Symposium on Electrical Insulation, Baltimore, MD, USA, 7-10 June 1992; pp. 161-164.

6. Li, H.; Li, J.; Ma, Y.; Yan, Q.; Ouyang, B. The role of thermo-oxidative aging at different temperatures on the crystal structure of crosslinked polyethylene. J. Mater. Sci. Mater. Electron. 2018, 29, 3696-3703. [CrossRef]

7. Paul, J.; Hansen, E.W.; Roots, J. Probing the molecular dynamics in XLPE aged at different temperatures by 1H NMR relaxation time measurements. Polym. Degrad. Stab. 2012, 97, 2403-2411. [CrossRef]

8. Montanari, G.C.; Motori, A. Thermal endurance evaluation of XLPE insulated cables. J. Phys. D Appl. Phys. 1991, 24, 1172-1181. [CrossRef]

9. Rasikawan, S.; Shimizu, N. Effect of additives on treeing initiation as a function of oxygen concentration in polyethylene. IEEE Trans. Electr. Insul. 1992, 27, 1089-1094. [CrossRef]

10. Mecheri, Y.; Nedjar, M.; Lamure, A.; Aufray, M.; Drouet, C. Influence of moisture on the electrical properties of XLPE insulation. In Proceedings of the 2010 Annual Report Conference on Electrical Insulation and Dielectric Phenomena, West Lafayette, IN, USA, 17-20 October 2010; pp. 1-4. [CrossRef]

11. Malik, N.H.; Al-Abdullah, A.A.; Al-Arainy, A.A.; Qureshi, M.I. Factors influencing electrical treeing in XLPE insulation. Eur. Trans. Electr. Power 2006, 16, 205-218. [CrossRef] 